\title{
POSITIVE SOLUTIONS FOR INFINITE SEMIPOSITONE/ POSITONE QUASILINEAR ELLIPTIC SYSTEMS WITH SINGULAR AND SUPERLINEAR TERMS
}

\author{
BRAHIM KHODJA AND ABDELKRIM MOUSSAOUI
}

Abstract. We establish existence and regularity of positive solutions for a class of quasilinear elliptic systems with singular and superlinear terms. The approach is based on sub-supersolution methods for systems of quasilinear singular equations and the Schauder's fixed point theorem.

Mathematics subject classification (2010): 35J75, 35J48, 35J92.

Keywords and phrases: singular systems, sub-supersolutions, Schauder's fixed point theorem, regularity.

\section{REFERENCES}

[1] C. O. Alves, F. J. S. A. CORRÊA, On the existence of positive solution for a class of singular systems involving quasilinear operators, Appl. Math. Comput., 185 (2007), 727-736.

[2] C. O. Alves, F. J. S. A. CorrêA, J. V. A. GonçAlves, Existence of solutions for some classes of singular Hamiltonian systems, Adv. Nonlinear Stud., 5 (2005), 265-278.

[3] H. BRÉZIS, Analyse fonctionnelle, theorie et applications, Masson, Paris, 1983.

[4] S. Carl, V. K. Le, D. Motreanu, Nonsmooth variational problems and their inequalities. Comparaison principles and applications, Springer, New York, 2007.

[5] S. El Manouni, K. Perera, R. Shivaji, On singular quasimonotone (p,q)-Laplacian systems, Proc. Roy. Soc. Edinburgh Sect. A, 142 (2012), 585-594.

[6] M. Ghergu, Lane-Emden systems with negative exponents, J. Functional Anal., 258 (2010), 3295 3318.

[7] M. Ghergu, Lane-Emden systems with singular data, Proc. Royal Society of Edinburgh: Section A (Math.) 141 (2011), 1279-1294.

[8] J. Giacomoni, J. HernandeZ, A. Mouss Aoui, Quasilinear and singular systems: the cooperative case, Contemporary Math., Amer. Math. Soc., Providence, R.I., 540 (2011), 79-94.

[9] J. Giacomoni, J. Hernandez, P. Sauvy, Quasilinear and singular elliptic systems, Adv. Nonl. Anal., 2 (2013), 1-41.

[10] J. Giacomoni, I. Schindler, P. TAKac, Sobolev versus Hölder local minimizers and existence of multiple solutions for a singular quasilinear equation, Ann. Sc. Norm. Super. Pisa Cl. Sci., 5, 6 (2007), 117-158.

[11] D. D. HAI, On a class of singular p-Laplacian boundary value problems, J. Math. Anal. Appl., 383 (2011), 619-626.

[12] J. Hernández, F.J. Mancebo, J.M. Vega, Positive solutions for singular semilinear elliptic systems, Adv. Diff. Eqts., 13 (2008), 857-880.

[13] E. K. Lee, R. ShivaJI, J. YE, Classes of singular pq-Laplacian semipositone systems, Disc. Contin. Dynam. Syst. A, 27 (2010), 1123-1132.

[14] E. K. LeE, R. ShivaJi, J. Ye, Classes of infinite semipositone systems, Diff. Integral Eqts., 24 (2011), 361-370.

[15] M. Montenegro, A. Suarez, Existence of a positive solution for a singular system, Proc. Roy. Soc. Edinburgh Sect. A, 140 (2010), 435-447. 
[16] D. Motreanu, V. V. Motreanu, N. Papageorgiou, Multiple constant sign and nodal solutions for nonlinear Neumann eigenvalue problems, Ann. Sc. Norm. Super. Pisa Cl. Sci., 5, 10 (2011), 729755.

[17] D. Motreanu, A. Moussadui, A quasilinear singular elliptic system without cooperative structure, Act. Math. Sci., 34 B, 3 (2014), 905-916.

[18] D. Motreanu, A. Mouss Aoui, Existence and boundedness of solutions for a singular cooperative quasilinear elliptic system, Complex Var. Elliptic Equ., 59 (2014), 285-296.

[19] D. Motreanu, A. Mouss aoui, An existence result for a class of quasilinear singular competitive elliptic systems, Applied Math. Letters, 38 (2014), 33-37.

[20] A. Mouss aoui, B. Khodja, S. TAs, A singular Gierer-Meinhardt system of elliptic equations in $R^{N}$, Nonl. Anal., 71 (2009), 708-716.

[21] H. YIN, Z. YANG, Existence and non-existence of entire positive solutions for quasilinear systems with singular and super-linear terms, Differ. Equ. Appl., 2, 2 (2010), 241-249.

[22] E. ZeIDLER, Nonlinear functional analysis and its applications. I. Fixed-point theorems, SpringerVerlag, New York, 1986. 